\title{
A new muth generated family of distributions with applica- tions
}

\author{
Abdullah M. Almarashia ${ }^{a}$ M. Elgarhy $y^{b, *}$ \\ a Statistics Department, Faculty of Science, King AbdulAziz University, Jeddah, Kingdom of Saudi Arabia. \\ ${ }^{b}$ Vice Presidency for Graduate Studies and Scientific Research, University of Jeddah, Jeddah, Kingdom of Saudi Arabia.
}

Communicated by R. Saadati

\begin{abstract}
A new family of distributions called the Muth family of distributions is introduced and studied. Five special submodels of the proposed family are discussed. Some mathematical properties of the Muth family are studied. Explicit expressions for the probability weighted, moments, mean deviation and order statistics are investigated. Maximum likelihood procedure is used to estimate the unknown parameters. One real data set is employed to show the usefulness of the new family.
\end{abstract}

Keywords: Muth distribution, Weibull distribution, moments, order statistics, maximum likelihood estimation.

2010 MSC: 60E05, 62E10, 62N05.

(C)2018 All rights reserved.

\section{Introduction}

The most popular traditional distributions often do not characterize and do not predict most of the interesting data sets. Generated family of continuous distributions is a new improvement for creating and extending the usual classical distributions. The newly generated families have been broadly studied in several areas as well as yield more flexibility in applications. Some well-established generators and other recently are the beta- $G$ by [10], gamma- $G$ by [18], Transformed-Transformer (T-X) by [3], Weibull-G by [4], exponentiated half-logistic-G by [6], the Type I half-logistic-G by [5], Garhy-G by [9], Kumaraswamy Weibull-G by [13], exponentiated Weibull-G by [12], Topp-Leone -G by [2], Type II half logistic-G by [14], generalized odd log-logistic by [7], and Odd Frechet-G by [11], among others.

Authors in [3] proposed T-X generated family of any continuous distribution with the following cdf

$$
\mathrm{F}(\mathrm{x})=1-\int_{0}^{-\log [\mathrm{G}(\mathrm{x} ; \zeta)]} \mathrm{r}(\mathrm{t}) \mathrm{dt} .
$$

In this new paper, we introduce a new generated family of distributions using the Muth distribution as a generator. This paper can be sorted as follows. In the next section, the Muth-generated (M-G) family

\footnotetext{
*Corresponding author

Email addresses: aalmarashi@kau.edu.sa (Abdullah M. Almarashi), m_elgarhy85@yahoo.com (M. Elgarhy)

doi: 10.22436/jnsa.011.10.06
} 
is defined. Section 3 concerns with some general mathematical properties of the family. In Section 4, five new special models of the generated family are considered. In Section 5, estimation of the parameters of the family is implemented through maximum likelihood method. Simulation study is carried out to estimate the model parameters of (MW) distribution in Section 6. An illustrative purpose on the basis of real data is investigated, in Section 7. Finally, concluding remarks are handled in Section 8.

\section{The new family}

Muth in 1977 introduced a continuous probability distribution with application in reliability theory [17]. A random variable $X$ is said to have a Muth distribution with parameter $\alpha$ if cumulative distribution function (cdf) is given by

$$
F(t)=1-e^{\alpha t-\frac{1}{\alpha}\left(e^{\alpha t}-1\right)}, 0<\alpha \leqslant 1 .
$$

The associated probability density function (pdf) is as follows

$$
f(t)=\left(e^{\alpha t}-\alpha\right) e^{\alpha t-\frac{1}{\alpha}\left(e^{\alpha t}-1\right)}, t>0 .
$$

Our goal here is to introduce a new family of distributions based on Muth pdf and the T-X family of [3]. The scope of the paper also includes study of the properties, estimation and applications of the evolved family of the distributions.

On the basis of cdf (1.1), we use the Muth distribution as a generator to obtain Muth generated family which is denoted by M-G. Hence the cdf of M-G family can be expressed as follows

$$
F(x)=1-\int_{0}^{-\log [G(x ; \zeta)]}\left(e^{\alpha t}-\alpha\right) e^{\alpha t-\frac{1}{\alpha}\left(e^{\alpha t}-1\right)} d t=C G(x ; \zeta)^{-\alpha} e^{-\frac{1}{\alpha} G(x ; \zeta)^{-\alpha}}, x \in R,
$$

where $(0<\alpha \leqslant 1)$ is a scale parameter, $C=e^{1 / \alpha}$ and $G(x ; \zeta)$ are the baseline cdf (with pdf $\left.g(x ; \zeta)\right)$ which may depend on the parameter $\zeta$. Therefore, the pdf of the Muth generated family is as follows

$$
f(x)=C g(x ; \zeta)\left(1-\alpha G(x ; \zeta)^{\alpha}\right) G(x ; \zeta)^{-2 \alpha-1} e^{-\frac{1}{\alpha} G(x, \zeta)^{-\alpha}}, x \in R .
$$

Hereafter, we denote by $X \sim M-G$ a random variable $X$ has pdf (2.2).

The survival function and hazard rate function are, respectively, given by

$$
\overline{\mathrm{F}}(x)=1-\mathrm{CG}(x ; \zeta)^{-\alpha} e^{-\frac{1}{\alpha} \mathrm{G}(x ; \zeta)^{-\alpha}}, x \in \mathrm{R},
$$

and

$$
h(x)=\frac{\operatorname{Cg}(x ; \zeta)\left(1-\alpha G(x ; \zeta)^{\alpha}\right) G(x ; \zeta)^{-2 \alpha-1} e^{-\frac{1}{\alpha} G(x ; \zeta)^{-\alpha}}}{1-C G(x ; \zeta)^{-\alpha} e^{-\frac{1}{\alpha} G(x ; \zeta)^{-\alpha}}} .
$$

\section{Some statistical properties}

This section provides some statistical properties of M-G family of distributions.

\subsection{Important representation}

Using exponential expansion,

$$
e^{-a x}=\sum_{i=0}^{\infty} \frac{(-1)^{i} a^{i} x^{i}}{i !}
$$

in pdf (2.2), we can write 


$$
f(x)=\operatorname{Cg}(x ; \zeta)\left(1-\alpha G(x ; \zeta)^{\alpha}\right) \sum_{i=0}^{\infty} \frac{(-1)^{i} G(x ; \zeta)^{-\alpha(i+2)-1}}{\alpha^{i} i !}, \quad x \in R .
$$

We can write the above equation as

$$
f(x)=\sum_{i=0}^{\infty} \eta_{i} g(x ; \zeta)\left(G(x ; \zeta)^{-\alpha(i+2)-1}-\alpha G(x ; \zeta)^{-\alpha(i+1)-1}\right), \quad x \in R,
$$

where,

$$
\eta_{i}=\frac{C \alpha^{-i}(-1)^{i}}{i !}
$$

By adding and subtracting one we can write equation as

$$
f(x)=\sum_{i=0}^{\infty} \eta_{i} g(x ; \zeta)\left((1-[1-G(x ; \zeta)])^{-\alpha(i+2)-1}-\alpha(1-[1-G(x ; \zeta)])^{-\alpha(i+1)-1}\right) .
$$

For $\mathrm{d}$, a positive real non integer and $|z|<1$, we have the generalized binomial series

$$
(1-z)^{-\mathrm{d}}=\sum_{j=0}^{\infty}\left(\begin{array}{c}
\mathrm{d}+j-1 \\
j
\end{array}\right) z^{j}
$$

and

$$
(1-z)^{\mathrm{d}}=\sum_{\mathrm{k}=0}^{\infty}(-1)^{\mathrm{k}}\left(\begin{array}{l}
\mathrm{d} \\
\mathrm{k}
\end{array}\right) z^{\mathrm{k}}
$$

Using the expansions (3.1) and (3.2) in the last pdf, we can write

$$
f(x)=\sum_{k=0}^{\infty} \eta_{k} g(x ; \zeta) G(x ; \zeta)^{k}, \quad x \in R,
$$

where,

$$
\eta_{k}=\sum_{i, j=0}^{\infty} \eta_{i}(-1)^{k}\left(\begin{array}{l}
j \\
k
\end{array}\right)\left[\left(\begin{array}{c}
\alpha(i+2)+j \\
j
\end{array}\right)-\alpha\left(\begin{array}{c}
\alpha(i+1)+j \\
j
\end{array}\right)\right] .
$$

Also, the pdf $f(x)$ of the M-G family can be expressed as follows

$$
f(x)=\sum_{k=0}^{\infty} W_{k} h_{k+1}(x),
$$

where, $W_{k}=\eta_{k} / k+1$, and $h_{a}(x)=a g(x ; \zeta) G(x ; \zeta)^{a-1}$. Equation (3.1) gives exponentiated-generated (exp-G) with power parameter $k$.

Also, for $h$, an integer, an expansion for the $[F(x)]^{h}$ is derived as follows

$$
[\mathrm{F}(\mathrm{x})]^{\mathrm{h}}=\sum_{z=0}^{\infty} \mathrm{s}_{z} \mathrm{G}(\mathrm{x} ; \zeta)^{z}
$$

where,

$$
s_{z}=\sum_{i, u=0}^{\infty} \frac{\mathrm{Ch}^{\mathrm{h}}(-1)^{\mathrm{i}+z} \mathrm{~h}^{z}}{\alpha^{z} z !}\left(\begin{array}{c}
\mathrm{u} \\
z
\end{array}\right)\left(\begin{array}{c}
\alpha(\mathrm{h}+\mathrm{i})+\mathrm{u}-1 \\
\mathrm{u}
\end{array}\right) .
$$

Density and cumulative functions (3.3) and (3.4) can be used to derive several mathematical properties of the M-G family. 


\subsection{The probability weighted moments}

Probability weighted moments (PWMs) of X cover the summarization and description of theoretical probability distributions. The elementary use of these moments is in the estimation of the parameters for a distribution whose inverse cannot be expressed explicitly. For a random variable $X$, the PWM, denoted by $\tau_{r, h}$, is defined by

$$
\tau_{r, h}=E\left[X^{r} F(x)^{h}\right]=\int_{-\infty}^{\infty} x^{r} f(x)(F(x))^{h} d x .
$$

Inserting (3.3) and (3.4) into (3.5), the PWM of M-G family is obtained as follows

$$
\tau_{r, h}=\int_{-\infty}^{\infty} \sum_{k, z=0}^{\infty} s_{z} \eta_{k} x^{r} g(x ; \zeta) G(x ; \zeta)^{k+z} d x .
$$

Then,

$$
\tau_{r, h}=\sum_{k, z=0}^{\infty} s_{z} \eta_{k} \tau_{r, k+z}
$$

\subsection{Moments}

The $r^{\text {th }}$ ordinary moment of $X$ follows from (3.3) as

$$
\mu_{r}^{\prime}=\int_{-\infty}^{\infty} x^{r} f(x) d x=\int_{-\infty}^{\infty} \sum_{k=0}^{\infty} \eta_{k} x^{r} g(x ; \zeta) G(x ; \zeta)^{k} d x
$$

Another formula of $r^{\text {th }}$ moment of $X$ is given by

$$
\mu_{r}^{\prime}=\sum_{k=0}^{\infty} \eta_{k} \tau_{r, k}
$$

where, $\tau_{r, k}$ is the PWM.

Furthermore, the moment generating function of $\mathrm{X}$ can be expressed as

$$
M_{X}(t)=\sum_{r=0}^{\infty} \frac{t^{r}}{r !} \mu_{r}^{\prime}=\sum_{r, k=0}^{\infty} \frac{t^{r}}{r !} \eta_{k} \tau_{r, k} .
$$

Additionally, the $\mathrm{n}^{\text {th }}$ incomplete moment of a distribution play an important role in the applications. Using (3.3), the $n^{\text {th }}$ incomplete moment of $X$ is given by

$$
K_{n}(y)=\int_{0}^{y} x^{n} f(x) d x=\int_{0}^{y} \sum_{k=0}^{\infty} \eta_{k} x^{n} g(x ; \zeta) G(x ; \zeta)^{k} d x .
$$

\subsection{The mean deviation}

For a random variable $X$ with pdf $f(x)$ and $\operatorname{cdf} F(x)$, the mean deviation about the mean and mean deviation about the median, are defined by

$$
\delta_{1}=2 \mu \mathrm{F}(\mu)-2 \mathrm{~T}(\mu) \quad \text { and } \quad \delta_{2}=\mu-2 \mathrm{~T}(\mathrm{M}),
$$

where, $\mu=E(X), M=\operatorname{Median}(X)$, and $T(q)=\int_{-\infty}^{q} x f(x) d x$ which is the first incomplete moment.

\subsection{Order statistics}

Order statistics have been extensively applied in many fields of statistics, such as reliability and life testing. Let $X_{1}, X_{2}, \ldots, X_{n}$ be independent and identically distributed (i.i.d) random variables with the corresponding continuous distribution function $F(x)$. Let $X_{1: n}<X_{2: n}<\cdots<X_{n: n}$ be the corresponding 
ordered statistics. The pdf of the $s^{\text {th }}$ order statistic is

$$
f_{s: n}(x)=-\frac{f(x)}{B(s, n-s+1)} \sum_{v=0}^{n-s}(-1)^{v}\left(\begin{array}{c}
n-s \\
v
\end{array}\right) F(x)^{v+s-1},
$$

where, $B(.,$.$) is the beta function. Inserting (3.1) and (3.3) in (3.4) and replacing h$ with $v+s-1$, the pdf of the $s^{\text {th }}$ order statistic for M-G distribution is

$$
f_{s: n}(x)=-\frac{g(x ; \zeta)}{B(s, n-s+1)} \sum_{\nu=0}^{n-s} \sum_{k, z=0}^{\infty} \eta_{k} p_{z, v} G(x ; \zeta)^{k+z}
$$

where $p_{z, v}=(-1)^{v}\left(\begin{array}{c}n-s \\ v\end{array}\right) s_{z}$.

Further, the $r^{\text {th }}$ moment of sthorder statistic for M-G family is defined by

$$
E\left(X_{s: n}^{r}\right)=\int_{-\infty}^{\infty} x^{r} f_{s: n}(x) d x
$$

Inserting (3.6) in (3.7), leads to

$$
E\left(X_{s: n}^{r}\right)=-\frac{1}{B(s, n-s+1)} \sum_{v=0}^{n-s} \sum_{k, z=0}^{\infty} \eta_{k} p_{z, v} \int_{-\infty}^{\infty} x^{r} g(x ; \zeta) G(x ; \zeta)^{k+z} d x
$$

Then,

$$
E\left(X_{s: n}^{r}\right)=-\frac{1}{B(s, n-s+1)} \sum_{\nu=0}^{n-s} \sum_{k, z=0}^{\infty} \eta_{k} p_{z, v} \tau_{r, k+z}
$$

\section{Special sub-models of the M-G family}

In this section, we discuss some special submodels of the M-G family of distributions, namely, Muth Uniform (MU), Muth Lomax (ML), Muth Rayleigh (MR), Muth exponential (ME), and Muth Weibull (MW) distributions. We also illustrate the flexibility of the proposed family by sketching the graphs for the pdf and hazard rate functions (hrf) of each special submodel mentioned above.

\subsection{Muth uniform distribution}

The Muth uniform (MU) model is defined from (2.1) by taking the cdf and pdf of the uniformly distributed random variable

$$
\mathrm{G}(x ; \zeta)=\frac{x}{\theta} \text { and } \mathrm{g}(x ; \zeta)=\frac{1}{\theta}, 0<x<\theta, \text { where } \zeta=(\theta)^{\mathrm{T}} .
$$

Then, the cdf of MU random variable is defined by

$$
F_{M U}(x)=C\left(\frac{x}{\theta}\right)^{-\alpha} \exp \left\{-\left(\frac{1}{\alpha}\right)\left(\left(\frac{x}{\theta}\right)^{-\alpha}\right)\right\}, \quad 0<x<\theta, 0<\alpha \leqslant 1 .
$$

The pdf and hrf of MU are given by

$$
\begin{aligned}
& f_{\mathrm{MU}}(x)=\frac{C}{\theta}\left(1-\alpha\left(\frac{x}{\theta}\right)^{\alpha}\right)\left(\frac{x}{\theta}\right)^{-2 \alpha-1} \exp \left\{-\left(\frac{1}{\alpha}\right)\left(\left(\frac{x}{\theta}\right)^{-\alpha}\right)\right\}, \\
& h_{\mathrm{MU}}(x)=\frac{\frac{C}{\theta}\left(1-\alpha\left(\frac{x}{\theta}\right)^{\alpha}\right)\left(\frac{x}{\theta}\right)^{-2 \alpha-1} \exp \left\{-\left(\frac{1}{\alpha}\right)\left(\left(\frac{x}{\theta}\right)^{-\alpha}\right)\right\}}{1-C\left(\frac{x}{\theta}\right)^{-\alpha} \exp \left\{-\left(\frac{1}{\alpha}\right)\left(\left(\frac{x}{\theta}\right)^{-\alpha}\right)\right\}} .
\end{aligned}
$$

Figure 1 sketchs the graphs for the pdf and hrf of the MU distribution for different parameter values. 

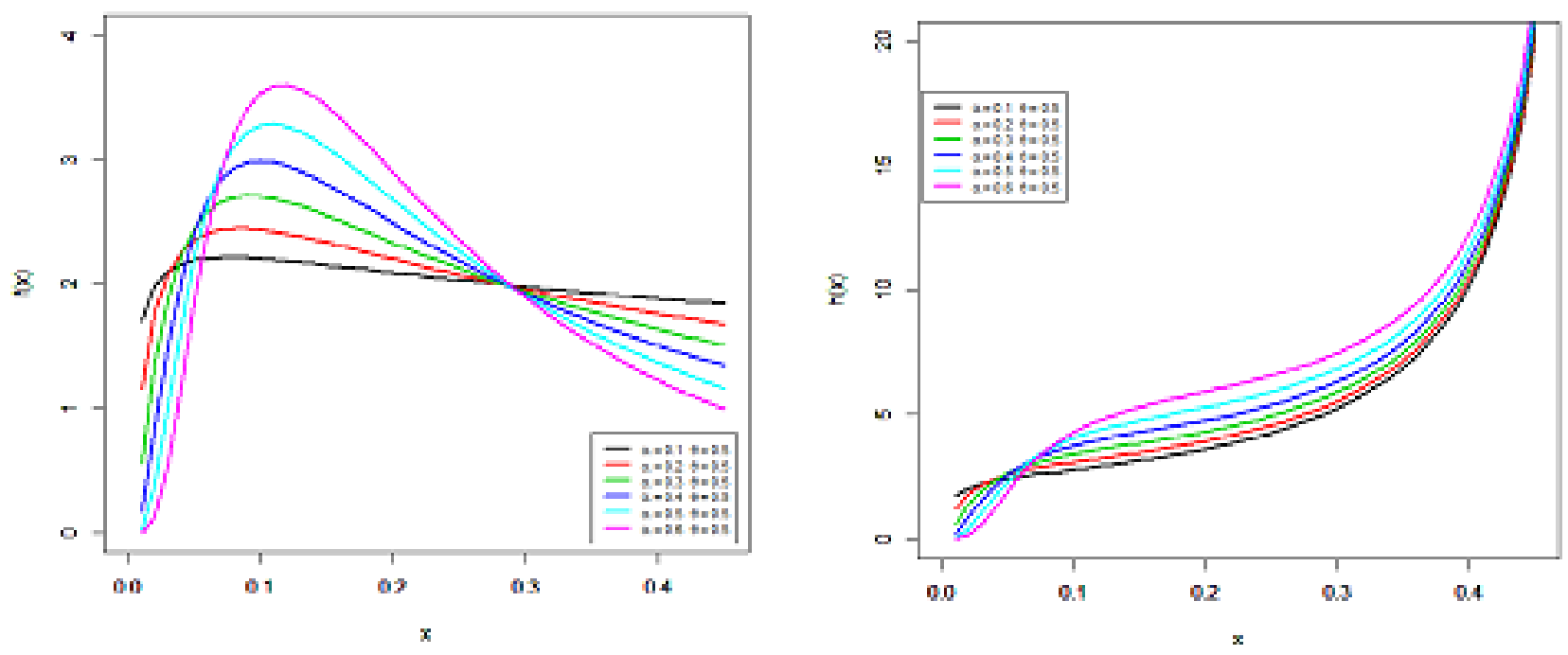

Figure 1: Pdf and hrf of MU distribution.

\subsection{Muth Lomax distribution}

A random variable $X$ is said to have a Lomax distribution, if its cdf and pdf are given by $G(x ; \zeta)=$ $1-(1+\lambda x)^{-\theta}$ and $g(x ; \zeta)=\theta \lambda(1+\lambda x)^{-(\theta+1)}$, where $\zeta=(\theta, \lambda)$. Then, the cdf of the Muth Lomax (ML) model is given by

$$
F_{M L}(x)=C\left(1-(1+\lambda x)^{-\theta}\right)^{-\alpha} e^{-\frac{1}{\alpha}\left(1-(1+\lambda x)^{-\theta}\right)^{-\alpha}}, \quad x, \theta, \lambda>0,0<\alpha \leqslant 1 .
$$

The pdf and hrf of ML distribution are expressed as

$$
\begin{aligned}
& f_{M L}(x)=C \theta \lambda(1+\lambda x)^{-(\theta+1)}\left(1-\alpha\left(1-(1+\lambda x)^{-\theta}\right)^{\alpha}\right)\left(1-(1+\lambda x)^{-\theta}\right)^{-2 \alpha-1} e^{-\frac{1}{\alpha}\left(1-(1+\lambda x)^{-\theta}\right)^{-\alpha},}, \\
& h_{M L}(x)=\frac{C \theta \lambda(1+\lambda x)^{-(\theta+1)}\left(1-\alpha\left(1-(1+\lambda x)^{-\theta}\right)^{\alpha}\right)\left(1-(1+\lambda x)^{-\theta}\right)^{-2 \alpha-1} e^{-\frac{1}{\alpha}\left(1-(1+\lambda x)^{-\theta}\right)^{-\alpha}}}{1-C\left(1-(1+\lambda x)^{-\theta}\right)^{-\alpha} e^{-\frac{1}{\alpha}\left(1-(1+\lambda x)^{-\theta}\right)^{-\alpha}}} .
\end{aligned}
$$

Figure 2 shows the pdf and hrf of the ML distribution for different parameter values.
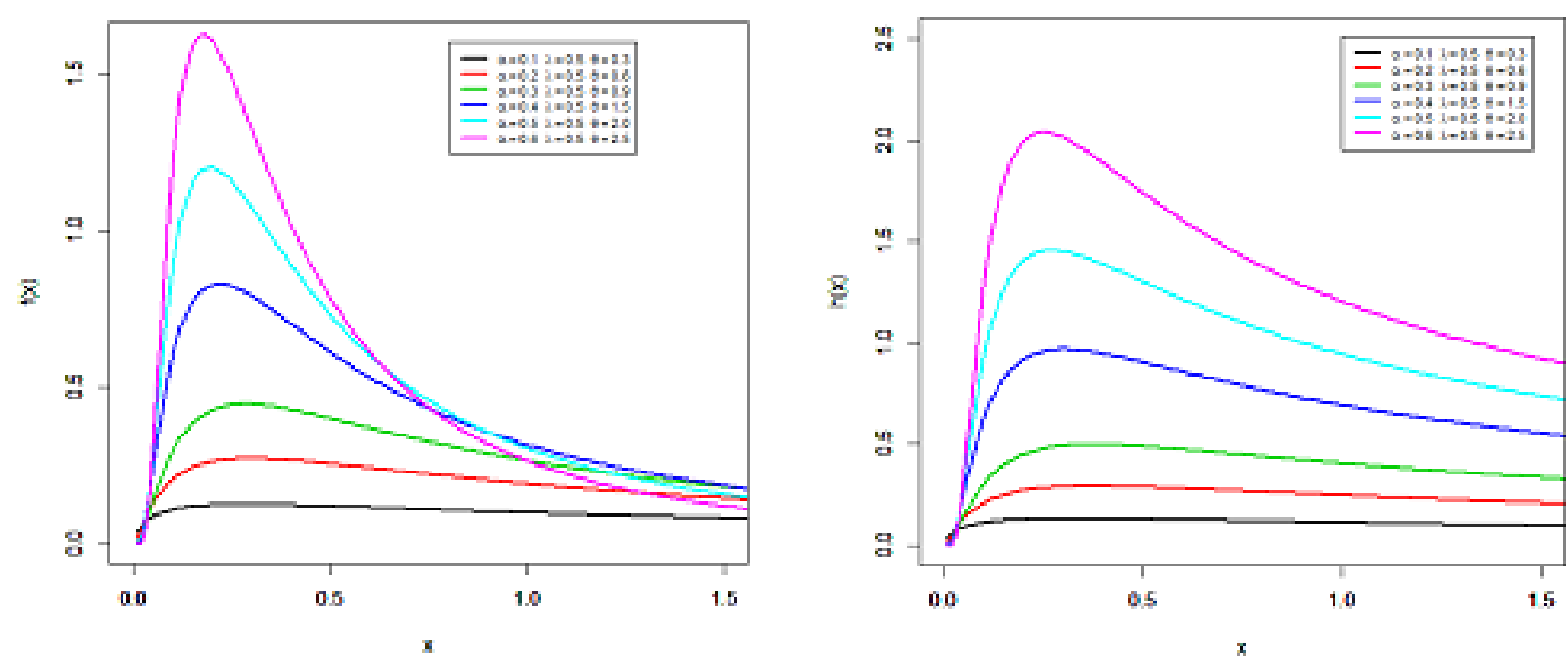

Figure 2: Pdf and hrf of ML distribution. 


\subsection{Muth Rayleigh distribution}

The cdf and density function of the Rayleigh random variable are expressed as $G(x ; \zeta)=1-e^{-\beta x^{2}}$ and $g(x ; \zeta)=2 \beta x e^{-\beta x^{2}}, x, \beta>0$, where $\zeta=(\beta)$. Then, the cdf of the MR distribution has the following form

$$
F_{M R}(x)=C\left(1-e^{-\beta x^{2}}\right)^{-\alpha} e^{-\frac{1}{\alpha}\left(1-e^{-\beta x^{2}}\right)^{-\alpha}}, \quad x, \beta>0,0<\alpha \leqslant 1 .
$$

The pdf and hrf of the MR model are

$$
\begin{aligned}
& f_{M R}(x)=C 2 \beta x e^{-\beta x^{2}}\left(1-\alpha\left(1-e^{-\beta x^{2}}\right)^{\alpha}\right)\left(1-e^{-\beta x^{2}}\right)^{-2 \alpha-1} e^{-\frac{1}{\alpha}\left(1-e^{-\beta x^{2}}\right)^{-\alpha}}, \\
& h_{M R}(x)=\frac{2 C \beta x e^{-\beta x^{2}}\left(1-\alpha\left(1-e^{-\beta x^{2}}\right)^{\alpha}\right)\left(1-e^{-\beta x^{2}}\right)^{-2 \alpha-1} e^{-\frac{1}{\alpha}\left(1-e^{-\beta x^{2}}\right)^{-\alpha}}}{1-C\left(1-e^{-\beta x^{2}}\right)^{-\alpha} e^{-\frac{1}{\alpha}\left(1-e^{-\beta x^{2}}\right)^{-\alpha}}} .
\end{aligned}
$$

Figure 3 displays the plots for the pdf and hrf of the MR distribution for selected values of the model parameters.
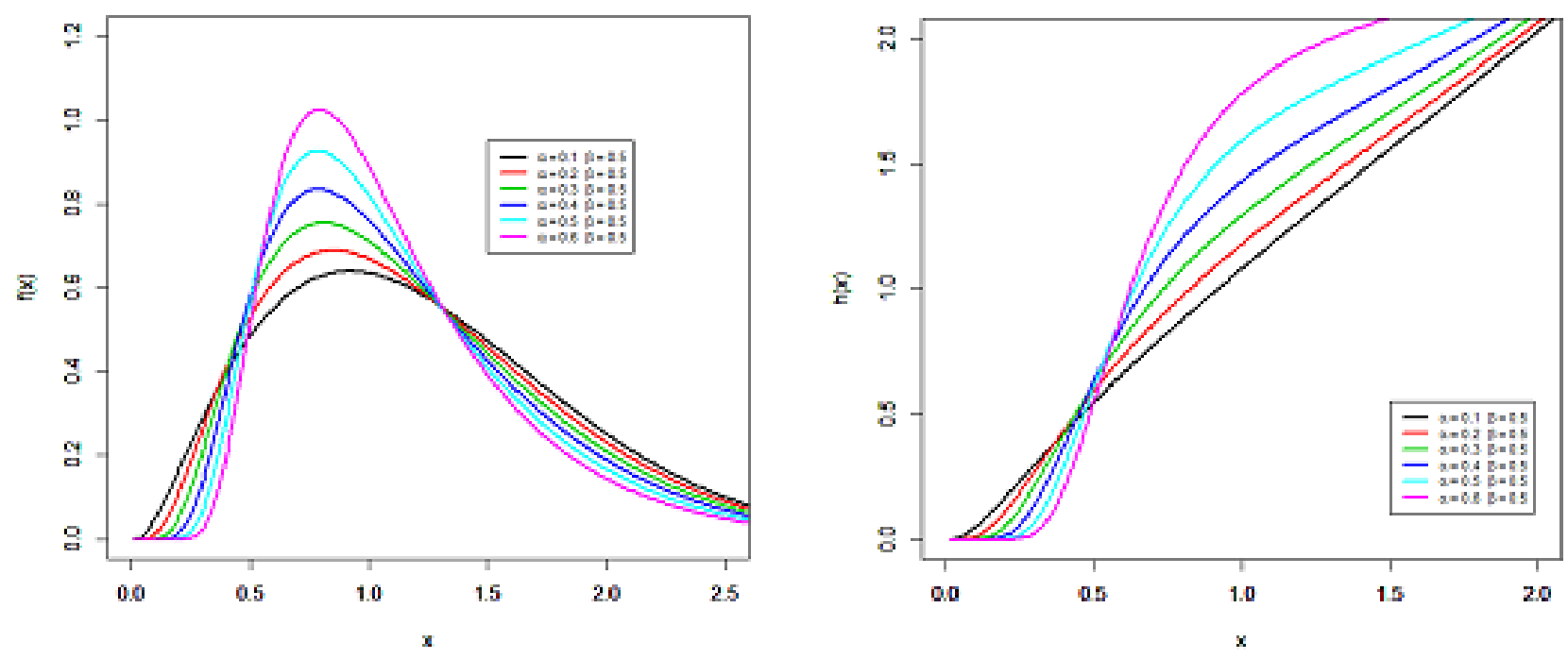

Figure 3: pdf and hrf of MR distribution.

\subsection{Muth exponential distribution}

The cdf and density function of the exponential random variable are expressed as $G(x ; \zeta)=1-e^{-\beta x}$ and $g(x ; \zeta)=\beta e^{-\beta x}, x, \beta>0$, where $\zeta=(\beta)$. Then, the cdf of the ME distribution has the following form

$$
F_{M E}(x)=C\left(1-e^{-\beta x}\right)^{-\alpha} e^{-\frac{1}{\alpha}\left(1-e^{-\beta x}\right)^{-\alpha}}, x, \beta>0,0<\alpha \leqslant 1 .
$$

The pdf and hrf of the ME model are

$$
\begin{aligned}
& f_{M E}(x)=C \beta e^{-\beta x}\left(1-\alpha\left(1-e^{-\beta x}\right)^{\alpha}\right)\left(1-e^{-\beta x}\right)^{-2 \alpha-1} e^{-\frac{1}{\alpha}\left(1-e^{-\beta x}\right)^{-\alpha}}, \\
& h_{M E}(x)=\frac{C \beta e^{-\beta x}\left(1-\alpha\left(1-e^{-\beta x}\right)^{\alpha}\right)\left(1-e^{-\beta x}\right)^{-2 \alpha-1} e^{-\frac{1}{\alpha}\left(1-e^{-\beta x}\right)^{-\alpha}}}{1-C\left(1-e^{-\beta x}\right)^{-\alpha} e^{-\frac{1}{\alpha}\left(1-e^{-\beta x}\right)^{-\alpha}}} .
\end{aligned}
$$

Figure 4 displays the plots for the pdf and hrf of the ME distribution for selected values of the model parameters. 

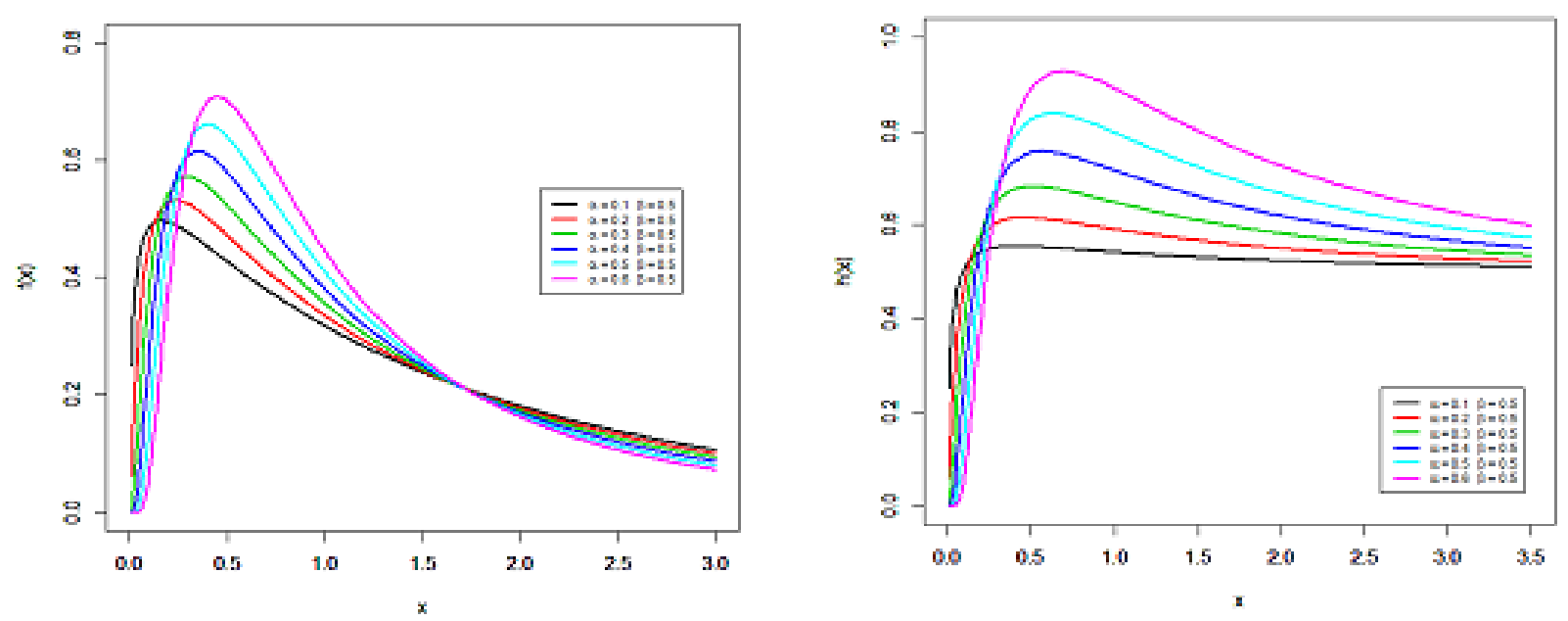

Figure 4: pdf and hrf of ME distribution.

\subsection{Muth Weibull distribution}

The cdf and density function of the Weibull random variable are expressed as $G(x ; \zeta)=1-e^{-\beta x^{\gamma}}$ and $g(x ; \zeta)=\gamma \beta x^{\gamma-1} e^{-\beta x^{\gamma}}, x, \beta, \gamma>0$, where $\xi=(\beta, \gamma)$. Then, the cdf of the MW distribution has the following form

$$
F_{M W}(x)=C\left(1-e^{-\beta x^{\gamma}}\right)^{-\alpha} e^{-\frac{1}{\alpha}\left(1-e^{-\beta x^{\gamma}}\right)^{-\alpha}}, x, \beta, \gamma>0,0<\alpha \leqslant 1 .
$$

The pdf and hrf of the MW model are

$$
\begin{aligned}
f_{M W}(x) & =C \gamma \beta x^{\gamma-1} e^{-\beta x^{\gamma}}\left(1-\alpha\left(1-e^{-\beta x^{\gamma}}\right)^{\alpha}\right)\left(1-e^{-\beta x^{\gamma}}\right)^{-2 \alpha-1} e^{-\frac{1}{\alpha}\left(1-e^{-\beta x^{\gamma}}\right)^{-\alpha}}, \\
h_{M W}(x) & =\frac{C \gamma \beta x^{\gamma-1} e^{-\beta x^{\gamma}}\left(1-\alpha\left(1-e^{-\beta x^{\gamma}}\right)^{\alpha}\right)\left(1-e^{-\beta x^{\gamma}}\right)^{-2 \alpha-1} e^{-\frac{1}{\alpha}\left(1-e^{-\beta x^{\gamma}}\right)^{-\alpha}}}{1-C\left(1-e^{-\beta x^{\gamma}}\right)^{-\alpha} e^{-\frac{1}{\alpha}\left(1-e^{-\beta x \gamma}\right)^{-\alpha}} .} .
\end{aligned}
$$

Figure 5 displays the plots for the pdf and hrf of the MW distribution for selected values of the model parameters.
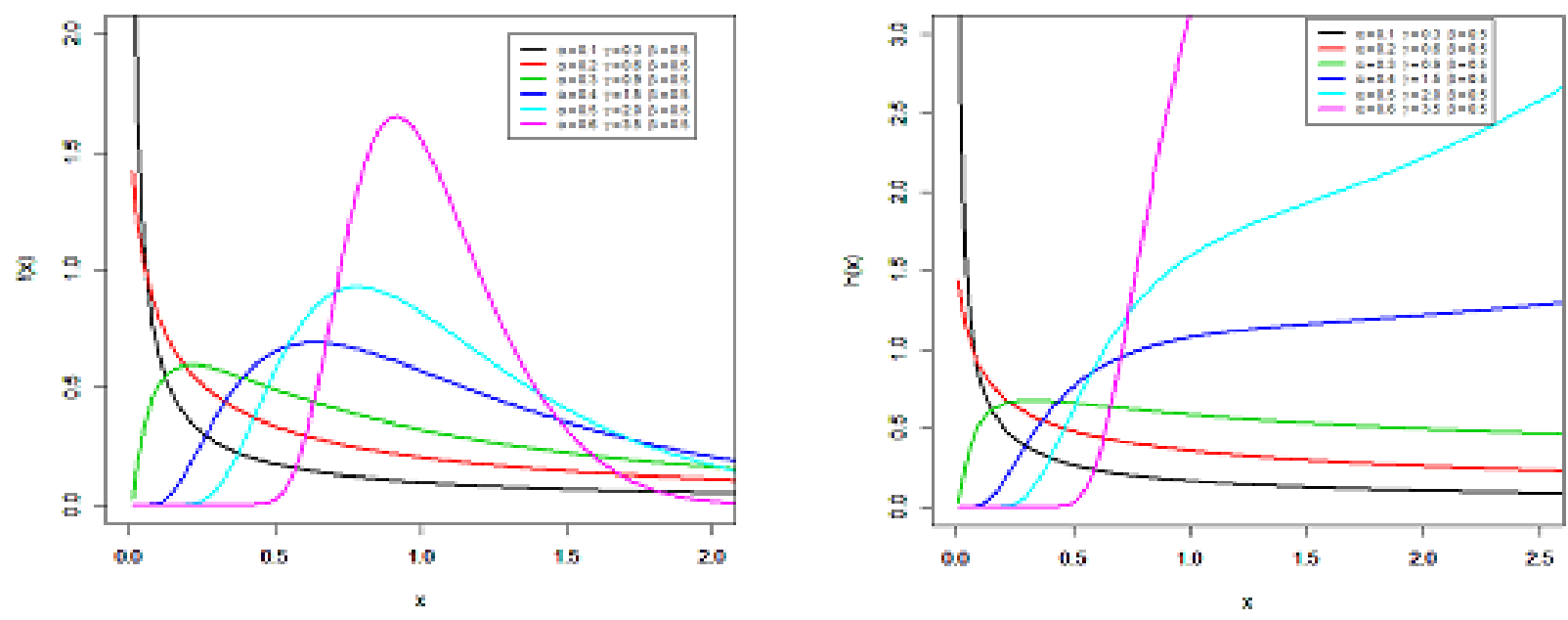

Figure 5: pdf and hrf of MW distribution.

\section{Maximum likelihood method}

We consider the estimation of the unknown parameters of M-G family from complete samples only by the method of maximum likelihood. Let $x_{1}, x_{2}, \ldots, x_{n}$ be the observed values from the M-G family with the parameter vector $\Phi=(\alpha, \zeta)^{\top}$. The log-likelihood function for $\Phi$ is given by 


$$
\ln L(\Phi)_{-}=\frac{n}{\alpha}+\sum_{i=1}^{n} \ln \left[g\left(x_{i} ; \zeta\right)\right]-(2 \alpha+1) \sum_{i=1}^{n} \ln \left[G\left(x_{i} ; \zeta\right)\right]+\sum_{i=1}^{n} \ln \left[1-\alpha G\left(x_{i} ; \zeta\right)^{\alpha}\right]-\frac{1}{\alpha} \sum_{i=1}^{n}\left[G\left(x_{i} ; \zeta\right)^{-\alpha}\right] .
$$

The elements of the score function $\mathrm{U}(\Phi)=\left(\mathrm{U}_{\alpha}, \mathrm{U}_{\zeta_{\mathrm{k}}}\right)$ are given by

$$
\mathrm{u}_{\alpha}=-\frac{\mathrm{n}}{\alpha^{2}}-2 \sum_{i=1}^{n} \ln \left[\mathrm{G}\left(x_{i} ; \zeta\right)\right]-\sum_{i=1}^{n} \frac{\mathrm{G}\left(x_{i} ; \zeta\right)^{\alpha}\left(1+\ln \mathrm{G}\left(x_{i} ; \zeta\right)\right)}{1-\alpha \mathrm{G}\left(x_{i} ; \zeta\right)^{\alpha}}+\frac{1}{\alpha^{2}} \sum_{i=1}^{n} \mathrm{G}\left(x_{i} ; \zeta\right)^{-\alpha}\left(1+\alpha \ln \mathrm{G}\left(x_{i} ; \zeta\right)\right)
$$

and

$$
\begin{aligned}
\mathrm{U}_{\zeta_{\mathrm{k}}}= & \sum_{i=1}^{n} \frac{\partial \mathrm{g}\left(x_{i} ; \zeta\right) / \partial \zeta_{\mathrm{k}}}{\mathrm{g}\left(x_{i} ; \zeta\right)}-(2 \alpha+1) \sum_{i=1}^{n} \frac{\partial \mathrm{G}\left(x_{i} ; \zeta\right) / \partial \zeta_{\mathrm{k}}}{\mathrm{G}\left(x_{i} ; \zeta\right)}-\alpha^{2} \sum_{i=1}^{n} \frac{\mathrm{G}\left(x_{i} ; \zeta\right)^{\alpha-1} \partial \mathrm{G}\left(x_{i} ; \zeta\right) / \partial \zeta_{\mathrm{k}}}{1-\alpha \mathrm{G}\left(x_{i} ; \zeta\right)^{\alpha}} \\
& +\sum_{i=1}^{n}\left[\mathrm{G}\left(x_{i} ; \zeta\right)^{-\alpha-1} \partial \mathrm{G}\left(x_{i} ; \zeta\right) / \partial \zeta_{k}\right] .
\end{aligned}
$$

Setting $\mathrm{U}_{\alpha}$ and $\mathrm{U}_{\zeta_{\mathrm{k}}}$ equal to zero and solving these equations simultaneously yield the maximum likelihood estimator $\hat{\Phi}=(\hat{\alpha}, \hat{\zeta})$ of $\Phi=(\alpha, \zeta)^{\mathrm{T}}$. These equations cannot be solved analytically and statistical software can be used to solve them numerically using iterative methods.

\section{Simulation}

It is very difficult to compare the theoretical performances of the different estimators (MLE) for the MW distribution. Therefore, simulation is needed to compare the performances of the estimation mainly with respect to their biases and mean square errors for different sample sizes. A numerical study is performed using Mathematica 9 software. Different sample sizes are considered through the experiments at sizes $n=20,30,50,100,200,300$. In addition, the different values of the parameters $\alpha, \beta$, and $\gamma$.

The experiment will be repeated 1000 times. In each experiment, the estimates of the parameters will be obtained by maximum likelihood method. The means, MSEs and biases for the different estimators will be reported from these experiments.

Table 1: The parameter estimation from MW distribution using MLE.

\begin{tabular}{|l|l|l|l|l|l|l|l|}
\hline \multirow{2}{*}{$\mathrm{n}$} & \multicolumn{2}{|c|}{ Set 1: $\alpha=0.1, \beta=0.5, \gamma=2$} & \multicolumn{3}{|c|}{ Set 2: $\alpha=0.1, \beta=0.5, \gamma=1.5$} \\
\cline { 2 - 8 } & Par & MLE & Bias & MSE & MLE & Bias & MSE \\
\hline \multirow{3}{*}{20} & $\alpha$ & 0.10009 & 0.00009 & $2.18542 \times 10^{-8}$ & 0.10009 & 0.00009 & $2.49495 \times 10^{-8}$ \\
\cline { 2 - 8 } & $\beta$ & 0.52885 & 0.02885 & 0.01616 & 0.52838 & 0.02837 & 0.01493 \\
\cline { 2 - 8 } & $\gamma$ & 2.35045 & 0.35045 & 1.06892 & 1.73074 & 0.23074 & 0.55934 \\
\hline \multirow{3}{*}{30} & $\alpha$ & 0.10009 & 0.00009 & $2.04174 \times 10^{-8}$ & 0.10009 & 0.00009 & $1.87626 \times 10^{-8}$ \\
\cline { 2 - 8 } & $\beta$ & 0.52298 & 0.02298 & 0.01014 & 0.51679 & 0.01679 & 0.01008 \\
\cline { 2 - 8 } & $\gamma$ & 2.23954 & 0.23954 & 0.67442 & 1.65002 & 0.15002 & 0.32692 \\
\hline \multirow{3}{*}{50} & $\alpha$ & 0.10009 & 0.00009 & $1.35184 \times 10^{-8}$ & 0.10010 & 0.00010 & $1.49952 \times 10^{-8}$ \\
\cline { 2 - 8 } & $\beta$ & 0.51326 & 0.01326 & 0.00557 & 0.50793 & 0.00793 & 0.00522 \\
\cline { 2 - 8 } & $\gamma$ & 2.13706 & 0.13706 & 0.29636 & 1.57555 & 0.07555 & 0.13456 \\
\hline \multirow{3}{*}{100} & $\alpha$ & 0.10009 & 0.00009 & $1.16819 \times 10^{-8}$ & 0.10009 & 0.00009 & $1.08969 \times 10^{-8}$ \\
\cline { 2 - 8 } & $\beta$ & 0.50590 & 0.00590 & 0.00272 & 0.50749 & 0.00749 & 0.00268 \\
\cline { 2 - 8 } & $\gamma$ & 2.05510 & 0.05510 & 0.12156 & 1.54784 & 0.04784 & 0.07201 \\
\hline \multirow{3}{*}{200} & $\alpha$ & 0.10009 & 0.00009 & $1.00063 \times 10^{-8}$ & 0.10009 & 0.00009 & $9.47676 \times 10^{-9}$ \\
\cline { 2 - 8 } & $\beta$ & 0.50310 & 0.00310 & 0.00126 & 0.50087 & 0.00087 & 0.00118 \\
\cline { 2 - 8 } & $\gamma$ & 2.03514 & 0.03514 & 0.06203 & 1.51971 & 0.01971 & 0.03250 \\
\hline \multirow{3}{*}{300} & $\alpha$ & 0.10009 & 0.00009 & $9.45489^{*} 10 \wedge-9$ & 0.10009 & 0.00009 & $9.54351 \times 10^{-9}$ \\
\cline { 2 - 7 } & $\beta$ & 0.50109 & 0.00109 & 0.0004 & 0.50146 & 0.00146 & 0.00079 \\
\cline { 2 - 7 } & $\gamma$ & 2.00758 & 0.00758 & 0.03576 & 1.51373 & 0.01373 & 0.02271 \\
\hline
\end{tabular}


Table 2: Continued of Table 1.

\begin{tabular}{|c|c|c|c|c|c|c|c|}
\hline \multirow[t]{2}{*}{$n$} & & \multicolumn{3}{|c|}{ Set 3: $\alpha=0.1, \beta=0.5, \gamma=2.5$} & \multicolumn{3}{|c|}{ Set 4: $\alpha=0.1, \beta=0.5, \gamma=3$} \\
\hline & Par & MLE & Bias & MSE & MLE & Bias & MSE \\
\hline \multirow{3}{*}{20} & $\alpha$ & 0.10009 & 0.00009 & $2.13806 \times 10^{-8}$ & 0.10009 & 0.00009 & $2.26347 \times 10^{-8}$ \\
\hline & $\beta$ & 0.52526 & 0.02526 & 0.01598 & 0.52575 & 0.02575 & 0.01605 \\
\hline & $\gamma$ & 2.88654 & 0.38654 & 1.48808 & 3.52130 & 0.52130 & 3.28233 \\
\hline \multirow{3}{*}{30} & $\alpha$ & 0.10009 & 0.00009 & $1.78784 \times 10^{-8}$ & 0.10009 & 0.00009 & $1.63314 \times 10^{-8}$ \\
\hline & $\beta$ & 0.51851 & 0.01851 & 0.00930 & 0.51467 & 0.01467 & 0.00925 \\
\hline & $\gamma$ & 2.78899 & 0.28899 & 0.95345 & 3.26266 & 0.26266 & 1.13238 \\
\hline \multirow{3}{*}{50} & $\alpha$ & 0.10009 & 0.00009 & $1.46953 \times 10^{-8}$ & 0.10009 & 0.00009 & $1.42012 \times 10^{-8}$ \\
\hline & $\beta$ & 0.50885 & 0.00885 & 0.00548 & 0.50721 & 0.00720 & 0.00537 \\
\hline & $\gamma$ & 2.62278 & 0.12278 & 0.40563 & 3.14215 & 0.14215 & 0.59788 \\
\hline \multirow{3}{*}{100} & $\alpha$ & 0.10009 & 0.00009 & $1.14768 \times 10^{-8}$ & 0.10009 & 0.00009 & $1.13114 \times 10^{-8}$ \\
\hline & $\beta$ & 0.50370 & 0.00370 & 0.00244 & 0.50519 & 0.00519 & 0.00269 \\
\hline & $\gamma$ & 2.56845 & 0.06845 & 0.18646 & 3.10045 & 0.10045 & 0.31007 \\
\hline \multirow{3}{*}{200} & $\alpha$ & 0.10009 & 0.00009 & $9.71289 \times 10^{-9}$ & 0.10009 & 0.00009 & $9.48756 \times 10^{-9}$ \\
\hline & $\beta$ & 0.50261 & 0.00261 & 0.00125 & 0.50430 & 0.00430 & 0.00121 \\
\hline & $\gamma$ & 2.53616 & 0.03616 & 0.08773 & 3.05770 & 0.05770 & 0.13228 \\
\hline \multirow{3}{*}{300} & $\alpha$ & 0.10009 & 0.00009 & $9.26478 \times 10^{-9}$ & 0.10009 & 0.00009 & $9.2883 \times 10^{-9}$ \\
\hline & $\beta$ & 0.50162 & 0.00162 & 0.00083 & 0.50096 & 0.00096 & 0.00083 \\
\hline & $\gamma$ & 2.52214 & 0.02214 & 0.05963 & 3.02670 & 0.02670 & 0.08808 \\
\hline
\end{tabular}

Table 3: Continued of Table 1

\begin{tabular}{|l|l|l|l|l|l|l|l|}
\hline $\mathrm{n}$ & & \multicolumn{7}{|c|}{ Set 5: $\alpha=0.1, \beta=0.7, \gamma=2$} & \multicolumn{3}{|c|}{ Set 6: $\alpha=0.1, \beta=0.7, \gamma=1.5$} \\
\cline { 2 - 8 } & Par & MLE & Bias & MSE & MLE & Bias & MSE \\
\hline \multirow{3}{*}{20} & $\alpha$ & 0.10009 & 0.00009 & $2.3686 \times 10^{-8}$ & 0.10010 & 0.00010 & $2.45403 \times 10^{-8}$ \\
\cline { 2 - 8 } & $\beta$ & 0.74089 & 0.04089 & 0.03260 & 0.73829 & 0.03829 & 0.03158 \\
\cline { 2 - 8 } & $\gamma$ & 2.24364 & 0.24364 & 0.81375 & 1.67011 & 0.17011 & 0.40653 \\
\hline \multirow{3}{*}{30} & $\alpha$ & 0.10009 & 0.00009 & $1.61678 \times 10^{-8}$ & 0.10009 & 0.00009 & $1.76236 \times 10^{-8}$ \\
\cline { 2 - 8 } & $\beta$ & 0.71937 & 0.01937 & 0.01880 & 0.72135 & 0.02135 & 0.01935 \\
\cline { 2 - 8 } & $\gamma$ & 2.18115 & 0.18115 & 0.50164 & 1.63293 & 0.13293 & 0.25457 \\
\hline \multirow{3}{*}{50} & $\alpha$ & 0.10009 & 0.00009 & $1.34529 \times 10^{-8}$ & 0.10009 & 0.00009 & $1.40571 \times 10^{-8}$ \\
\cline { 2 - 8 } & $\beta$ & 0.71340 & 0.01340 & 0.01058 & 0.71155 & 0.01155 & 0.01100 \\
\cline { 2 - 8 } & $\gamma$ & 2.09565 & 0.09565 & 0.22241 & 1.56544 & 0.06544 & 0.12047 \\
\hline \multirow{3}{*}{100} & $\alpha$ & 0.10009 & 0.00009 & $1.15168 \times 10^{-8}$ & 0.10009 & 0.00009 & $1.07646 \times 10^{-8}$ \\
\cline { 2 - 8 } & $\beta$ & 0.70765 & 0.00765 & 0.00530 & 0.70608 & 0.00608 & 0.00579 \\
\cline { 2 - 8 } & $\gamma$ & 2.04850 & 0.04850 & 0.10132 & 1.53947 & 0.03947 & 0.05280 \\
\hline \multirow{3}{*}{200} & $\alpha$ & 0.10009 & 0.00009 & $9.9711 \times 10^{-9}$ & 0.10009 & 0.00009 & $9.88463 \times 10^{-9}$ \\
\cline { 2 - 8 } & $\beta$ & 0.70320 & 0.00320 & 0.00234 & 0.70452 & 0.00452 & 0.00253 \\
\cline { 2 - 7 } & $\gamma$ & 2.02366 & 0.02366 & 0.04718 & 1.51997 & 0.01997 & 0.02705 \\
\hline \multirow{3}{*}{300} & $\alpha$ & 0.10009 & 0.00009 & $9.37211 \times 10^{-9}$ & 0.10009 & 0.00009 & $9.49605 \times 10^{-9}$ \\
\cline { 2 - 7 } & $\beta$ & 0.70173 & 0.00173 & 0.00170 & 0.70170 & 0.00170 & 0.00164 \\
\cline { 2 - 7 } & $\gamma$ & 2.01799 & 0.01799 & 0.03385 & 1.51232 & 0.01232 & 0.01812 \\
\hline
\end{tabular}


Table 4: Continued of Table 1.

\begin{tabular}{|l|l|l|l|l|l|l|l|}
\hline \multirow{2}{*}{$n$} & & Set 7: $\alpha=0.1, \beta=0.7, \gamma=2.5$ & \multicolumn{2}{|c|}{ Set 8: $\alpha=0.1, \beta=0.7, \gamma=3$} \\
\cline { 2 - 8 } & Par & MLE & Bias & MSE & MLE & Bias & MSE \\
\hline \multirow{3}{*}{20} & $\alpha$ & 0.10009 & 0.00009 & $2.16284 \times 10^{-8}$ & 0.10009 & 0.00009 & $2.15409 \times 10^{-8}$ \\
\cline { 2 - 8 } & $\beta$ & 0.72999 & 0.02999 & 0.03208 & 0.73549 & 0.03549 & 0.03269 \\
\cline { 2 - 8 } & $\gamma$ & 2.78802 & 0.28802 & 1.20123 & 3.35507 & 0.35507 & 1.81402 \\
\hline \multirow{3}{*}{30} & $\alpha$ & 0.10010 & 0.00010 & $1.95963 \times 10^{-8}$ & 0.10009 & 0.00009 & $1.77599 \times 10^{-8}$ \\
\cline { 2 - 8 } & $\beta$ & 0.72348 & 0.02348 & 0.01969 & 0.72194 & 0.02194 & 0.01908 \\
\cline { 2 - 8 } & $\gamma$ & 2.70308 & 0.20308 & 0.64998 & 3.24113 & 0.24113 & 0.92983 \\
\hline \multirow{3}{*}{50} & $\alpha$ & 0.10009 & 0.00009 & $1.48897 \times 10^{-8}$ & 0.10009 & 0.00009 & $1.34612 \times 10^{-8}$ \\
\cline { 2 - 8 } & $\beta$ & 0.71357 & 0.01357 & 0.01018 & 0.71520 & 0.01520 & 0.01093 \\
\cline { 2 - 8 } & $\gamma$ & 2.61487 & 0.11487 & 0.35466 & 3.16956 & 0.16956 & 0.54674 \\
\hline \multirow{3}{*}{100} & $\alpha$ & 0.10009 & 0.00009 & $1.13358 \times 10^{-8}$ & 0.10009 & 0.00009 & $1.15164 \times 10^{-8}$ \\
\cline { 2 - 8 } & $\beta$ & 0.71081 & 0.01081 & 0.00527 & 0.70453 & 0.00453 & 0.00517 \\
\cline { 2 - 8 } & $\gamma$ & 2.58545 & 0.08545 & 0.16481 & 3.08678 & 0.08678 & 0.23369 \\
\hline \multirow{3}{*}{200} & $\alpha$ & 0.10009 & 0.00009 & $9.86193 \times 10^{-9}$ & 0.10009 & 0.00009 & $9.79476 \times 10^{-9}$ \\
\cline { 2 - 8 } & $\beta$ & 0.70473 & 0.00473 & 0.00251 & 0.70372 & 0.00372 & 0.00238 \\
\cline { 2 - 8 } & $\gamma$ & 2.54455 & 0.04455 & 0.07743 & 3.04451 & 0.04451 & 0.10685 \\
\hline \multirow{3}{*}{300} & $\alpha$ & 0.10009 & 0.00009 & $9.56165 \times 10^{-9}$ & 0.10009 & 0.00009 & $9.39515 \times 10^{-9}$ \\
\cline { 2 - 8 } & $\beta$ & 0.70245 & 0.00245 & 0.00181 & 0.70333 & 0.00333 & 0.00163 \\
\cline { 2 - 7 } & $\gamma$ & 2.51537 & 0.01537 & 0.04910 & 3.02654 & 0.02654 & 0.06716 \\
\hline
\end{tabular}

\section{Real life applications}

In this section, we use one real data set to illustrate the importance and flexibility of the MW distribution. We compare the fits of the MW model with some models namely: the beta Weibull (BW) [15], Mcdonald Weibull (McW) [8], and exponentiated Weibull (EW) [16] distributions.

The maximized log-likelihood $(-2 \ell)$, Akaike information criterion (AIC), the corrected Akaike information criterion (CAIC), Bayesian information criterion (BIC), Hannan-Quinn information criterion (HQIC), Anderson-Darling $(A *)$, and Cramér-Von Mises $(W *)$ statistics are used for model selection.

The data set is obtained from studies in [1] and represents failure times of 84 Aircraft Windshield. The data are summarized in Table 5:

Table 5: The failure times aircraft windshield.

\begin{tabular}{|c|l|l|}
\hline \multirow{5}{*}{ Data Set } & $0.040,1.866,2.385,3.443,0.301,1.876,2.481,3.467,0.309,1.899,2.610,3.478,0.557,1.911$, \\
& $2.625,3.578,0.943,1.912,2.632,3.595,1.070,1.914,2.646,3.699,1.124,1.981,2.661,3.779$, \\
& $1.248,2.010,2.688,3.924,1.281,2.038,2.82,3,4.035,1.281,2.085,2.890,4.121,1.303,2.089$, \\
& $2.902,4.167,1.432,2.097,2.934,4.240,1.480,2.135,2.962,4.255,1.505,2.154,2.964,4.278$, \\
& $1.506,2.190,3.000,4.305,1.568,2.194,3.103,4.376,1.615,2.223,3.114,4.449,1.619,2.224$, \\
& $3.117,4.485,1.652,2.229,3.166,4.570,1.652,2.300,3.344,4.602,1.757,2.324,3.376,4.663$ \\
\hline
\end{tabular}

For the data set, Table 6 gives the MLEs of the fitted models and their standard errors (SEs) in parenthesis. The values of goodness-of-fit statistics are listed in Table 7.

It is noted, from Table 7, that the MW distribution provides a better fit than other competitive fitted models. It has the smallest values for goodness-of-fit statistics among all fitted models. Plots of the histogram, fitted densities and estimated cdfs are shown in Figures 6 and 7, respectively. These figures supported the conclusion drawn from the numerical values in Table 7.

\begin{tabular}{|l|l|} 
Table 6: The MLEs and SEs for the data set. \\
\begin{tabular}{|l|l|l|l|l|l|}
\hline Model & \multicolumn{7}{|c|}{ Estimates (SEs) } \\
\hline $\operatorname{MW}(\alpha, \beta, \gamma)$ & $2.44 \times 10^{-3}(0.0539)$ & $0.151(0.022)$ & $1.863(0.118)$ & & \\
\hline $\operatorname{BW}(\mathrm{a}, \mathrm{b}, \lambda, \gamma)$ & $53.874(2.717)$ & $20.528(0.278)$ & $1.076(0.278)$ & $0.231(0.184)$ & \\
\hline $\operatorname{McW}(\mathrm{a}, \mathrm{b}, \lambda, \gamma, \mathrm{c})$ & $51.321(5.329)$ & $19.762(0.605)$ & $1.119(0.48)$ & $0.23(0.424)$ & $1.525(38.539)$ \\
\hline $\operatorname{EW}(\lambda, \gamma, \mathrm{a})$ & $7.017(0.134)$ & $0.144(0.063)$ & $1773(0.827)$ & & \\
\hline
\end{tabular}
\end{tabular}




Table 7: Goodness-of-Fit statistics for the data set.
\begin{tabular}{|c|c|c|c|c|c|c|c|}
\hline Model & $-2 \ell$ & AIC & CAIC & BIC & HQIC & A* & W* \\
\hline MW & 266.952 & 272.952 & 273.252 & 272.724 & 275.883 & 0.78033 & 0.0653 \\
\hline BW & 289.948 & 297.948 & 298.455 & 297.645 & 301.857 & 3.34711 & 0.48715 \\
\hline McW & 283.983 & 293.983 & 294.752 & 293.604 & 298.869 & 3.33313 & 0.4847 \\
\hline EW & 320.347 & 326.347 & 326.647 & 324.196 & 326.302 & 32.74879 & 7.04167 \\
\hline
\end{tabular}

It is observed, from Table 7, that the MW distribution gives a better fit than other fitted models. Plots of the histogram, fitted densities and estimated cdfs are displayed in Figures 6 and 7, respectively. Figure 8 displays the pp plots of the MW distribution and other competitive models.

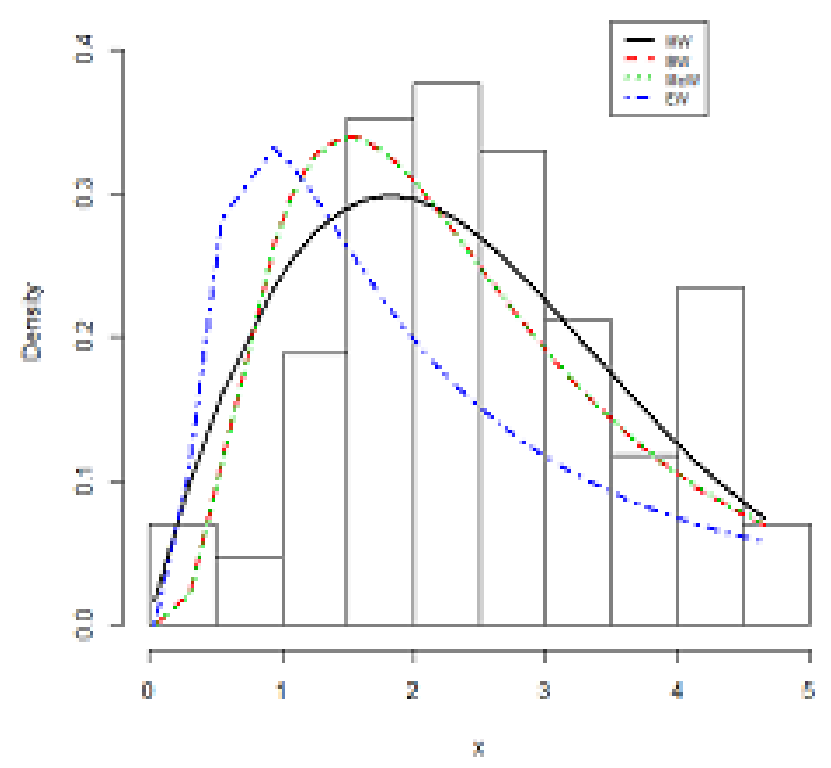

Figure 6: Estimated pdfs for the data Set.

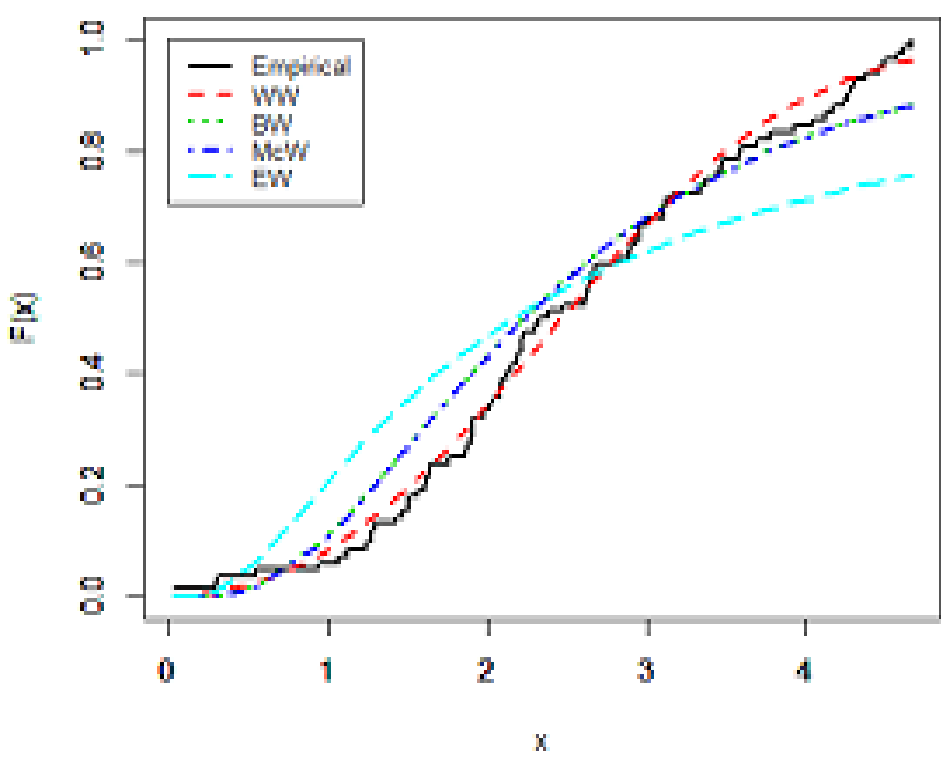

Figure 7: Estimated cdfs for the data set. 

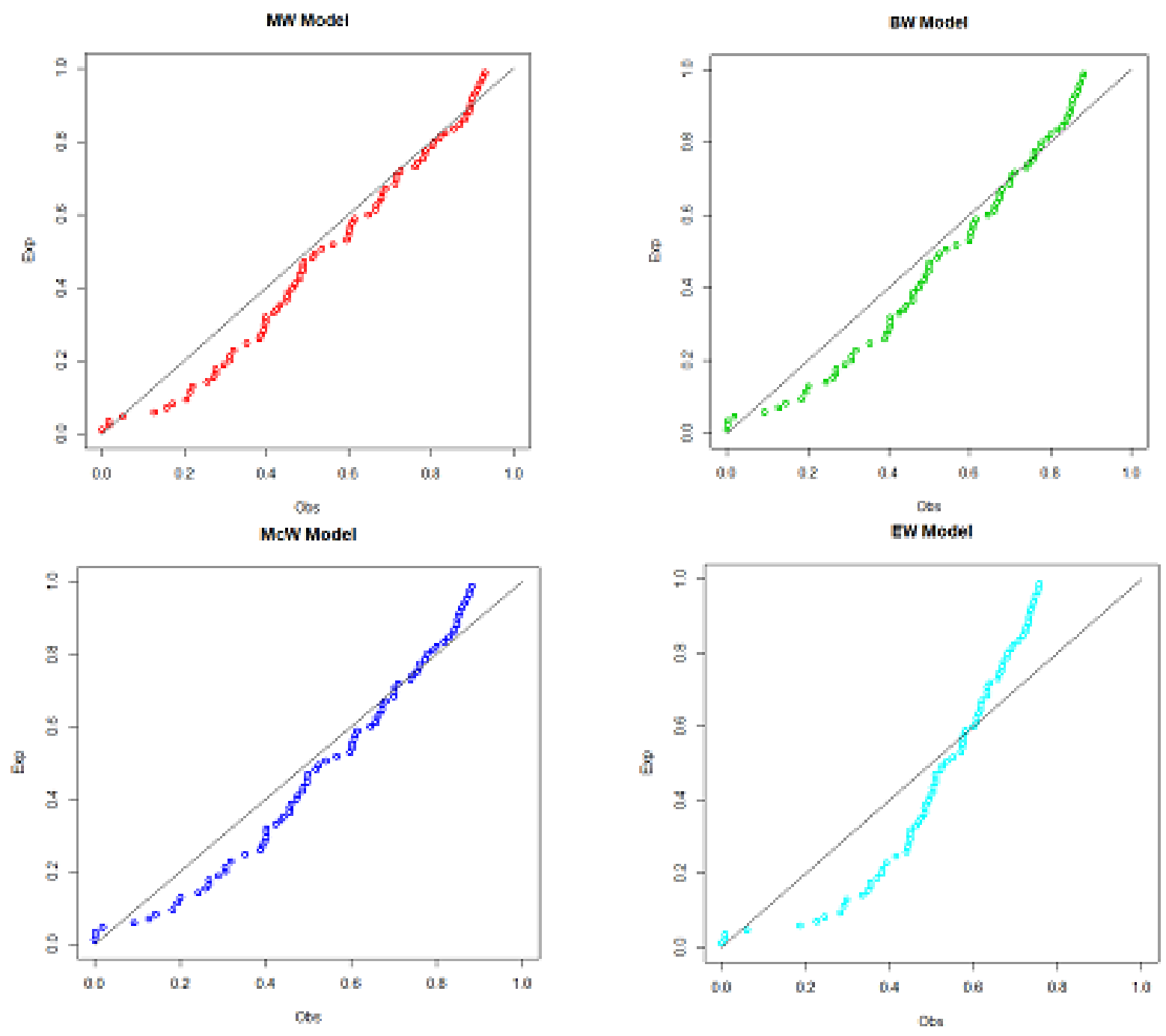

Figure 8: pp plots of the MW model and other models for the data set.

\section{Conclusion}

In this paper we propose a new class of distributions called the Muth-G family. Five special submodels are presented. We investigate several structural properties of the new distribution such as expansion for the density and cumulative functions and explicit expressions for the ordinary and incomplete moments, generating function, mean deviation, and order statistics. We estimate the parameters using maximum likelihood method. We perform a Monte Carlo simulation study for one particular case to assess the finite sample behavior of the maximum likelihood estimators. Applications to real data prove empirically the importance and potentiality of the suggested family.

\section{Acknowledgment}

This work was supported by the Deanship of Scientific Research (DSR), King AbdulAziz University, Jeddah, under grant No. (D-148-130-1439). The authors, therefore, gratefully acknowledge the DSR technical and financial support.

\section{References}

[1] T. H. M. Abouelmagd, S. Al-mualim, M. Elgarhy, A. Z. Afify, M. Ahmad, Properties of the four-parameter Weibull distribution and its Applications, Pakistan J. Statist., 33 (2017), 449-466. 7 
[2] A. Al-Shomrani, O. Arif, A. Shawky, S. Hanif, M. Q. Shahbaz, Topp-Leone family of distributions: Some properties and application, Pak. J. Stat. Oper. Res., 12 (2016), 443-451. 1

[3] A. Alzaatreh, C. Lee, F. Famoye, A new method for generating families of continuous distributions, Metron, 71 (2013), 63-79. 1, 2

[4] M. Bourguignon, R. B. Silva, G. M. Cordeiro, The Weibull-G family of probability distributions, J. Data Sci., 12 (2014), 53-68. 1

[5] G. M. Cordeiro, M. Alizadeh, P. R. D. Marinho, The type I half-logistic family of distributions, J. Stat. Comput. Simul., 86 (2016), 707-728. 1

[6] G. M. Cordeiro, M. Alizadeh, E. M. M. Ortega, The exponentiated half-logistic family of distributions: properties and applications, J. Probab. Stat., 2014 (2014), 21 pages. 1

[7] G. M. Cordeiro, M. Alizadeh, G. Ozel, B. Hosseini, E. M. M. Ortega, E. Altun, The generalized odd log-logistic family of distributions: properties, regression models and applications, J. Stat. Comput. Simul., 87 (2017), 908-932. 1

[8] G. M. Cordeiro, E. M. Hashimoto, E. M. M. Ortega, The McDonald Weibull model, Statistics, 48 (2014), 256-278. 7

[9] M. Elgarhy, A. S. Hassan, M. Rashed, Garhy-generated family of distributions with application, Math. Theory Model., 6 (2016), 1-15. 1

[10] N. Eugene, C. Lee, F. Famoye, Beta-normal distribution and its applications, Comm. Statist. Theory Methods, 31 (2002), 497-512. 1

[11] M. A. Haq, M. Elgarhy, The odd Frchet-G family of probability distributions, J. Stat. Appl. Prob., 7 (2018), 185-201. 1

[12] A. S. Hassan, M. Elgarhy, A New Family of Exponentiated Weibull-Generated Distributions, Int. J. Math. Appl., 4 (2016), 135-148. 1

[13] A. S. Hassan, M. Elgarhy, Kumaraswamy Weibull-generated family of distributions with applications, Adv. Appl. Statist., 48 (2016), 205-239. 1

[14] A. S. Hassan, M. Elgarhy, M. Shakil, Type II half Logistic family of distributions with applications, Pak. J. Stat. Oper. Res., 13 (2017), 245-264. 1

[15] C. Lee, F. Famoye, O. Olumolade, Beta-Weibull distribution: some properties and applications to censored data, J. Mod. Appl. Stat. Meth., 6 (2007), 173-186. 7

[16] G. S. Mudholkar, D. K. Srivastava, Exponentiated Weibull family for analysing bathtub failure rate data, IEEE T. Reliab., 42 (1993), 299-302. 7

[17] J. E. Muth, Reliability models with positive memory derived from the mean residual life function, C.P. Tsokos, I. N. Shimi (Eds.), Theory and Applications of Reliability, Academic Press, (1977), 401-434. 2

[18] M. M. Ristic, N. Balakrishnan, The gamma-exponentiated exponential distribution, J. Stat. Comput. Simul., 82 (2012), 1191-1206. 1 